\title{
Alternative approach to thermodynamic phase transitions
}

\author{
Seyed Hossein Hendi ${ }^{1,2 *}$, Shahram Panahiyan ${ }^{1,3,4} \dagger$, Behzad Eslam Panah ${ }^{1,2,5 \ddagger}$ and Mubasher Jamil ${ }^{6,7 \S}$ \\ 1 Physics Department and Biruni Observatory, College of Sciences, Shiraz University, Shiraz 71454, Iran \\ ${ }^{2}$ Research Institute for Astronomy and Astrophysics of Maragha (RIAAM), P. O. Box 55134-441, Maragha, Iran \\ ${ }^{3}$ Physics Department, Shahid Beheshti University, Tehran 19839, Iran \\ 4 Helmholtz-Institut Jena, Fröbelstieg 3, Jena 07743, Germany \\ 5 ICRANet, Piazza della Repubblica 10, I-65122 Pescara, Italy \\ ${ }^{6}$ School of Natural Sciences (SNS), National University of Sciences and Technology, H-12, Islamabad, 44000, Pakistan \\ 7 Institute for Astrophysics, Zhejiang University of Technology, Hangzhou, China
}

\begin{abstract}
One of the major open problems in theoretical physics is a consistent quantum gravity theory. Recent developments in thermodynamic phase transitions of black holes and their van der Waalslike behavior may provide an interesting quantum interpretation of classical gravity. Studying different methods of investigating phase transitions can extend our insight into the nature of quantum gravity. In this paper, we present an alternative theoretical approach for finding thermodynamic phase transitions in the extended phase space. Unlike the standard methods based on the usual equation of state involving temperature, our approach uses a new quasi-equation constructed from the slope of temperature versus entropy. This approach addresses some of the shortcomings of the other methods, and provides a simple and powerful way of studying the critical behavior of a thermodynamical system. Among the applications of this approach, we emphasize the analytical demonstration of possible phase transition points, and the identification of the non-physical range of horizon radii for black holes.
\end{abstract}

\section{INTRODUCTION}

Phase transitions are one of the interesting and challenging experimental and theoretical topics in various areas of science, from biological [1] to physical systems [2]. Regarding the physical systems, one may find that phase transitions play an important role in elementary particle physics [3] , condensed matter [4 6], the usual thermodynamics [7], cosmology [8], black holes [9] and other branches of physics. In general, there are at least three well-known approaches to discuss phase transitions inside a black hole. Two of these approaches are based on a macroscopic point of view (Davies and LandauLifshitz methods that discuss, respectively, the behavior of heat capacity and thermodynamic fluctuations), and one of them is based on a microscopic viewpoint (thermodynamic geometry or Ruppeiner geometry). In this regard, we focus on the thermodynamic phase transition of black holes. There are several well-known approaches for studying the critical behavior of black holes. One of them uses heat capacity in the context of canonical ensemble. The discontinuity of heat capacity is where the phase transition takes place. Another method is based on studying the van der Waals-like behavior of black holes in the extended phase space by considering a proportionality between the cosmological constant and dynamical pressure. This method is based on an equation of state, which originates from temperature. For details, please re-

\footnotetext{
*email address: hendi@shirazu.ac.ir

$\dagger$ email address: shahram.panahiyan@uni-jena.de

$\ddagger$ email address: behzad.eslampanah@gmail.com

$\S$ email address: mjamil@zjut.edu.cn
}

fer to earlier reviews on phase transitions of black holes, black rings, black saturns and black membranes [10 13], where classical instability and horizon topology changing transitions are also discussed.

In this paper, we consider the method for extracting the critical values and the van der Waals phase transition based on the divergence of heat capacity (the Davies method). We should note that our method is applicable in the extended phase space thermodynamics of black hole, in which the cosmological constant is considered as dynamic pressure. Due to the fact that in our approach we use the behavior of the heat capacity, it is not a novel approach, but an alternative way for obtaining the properties of heat capacity based on the canonical ensemble. In other words, this method is the same as the Davies method, but with a different point of view. We introduce an alternative approach for obtaining the critical values of the van der Waals-like behavior by using the slope of $T$ versus $S$, and give two relevant examples. In other words, in the usual extended phase space, one calculates $T=\left(\frac{\partial M}{\partial S}\right)$ to obtain an equation of state. Other calculations and interpretations are based on such an equation of state. In our approach, we use $\left(\frac{\partial T}{\partial S}\right)=\left(\frac{\partial^{2} M}{\partial S^{2}}\right)=0$ to obtain a new relation for pressure. This relation is free of temperature, and therefore, it is not the usual equation of state. One finds that this relation, and other related quantities and phase diagrams, are different from those obtained in the usual extended phase space. Our method provides the possibility of mapping all phase transition points in a system. In addition, it can single out the non-physical range of horizon radii in which the black hole solutions do not exist. Furthermore, the rate of increase of non-physical range of horizon radii for different critical values can be obtained. As a final point, 
we should note that our approach is quite different from the Poincare's turning point method [14 17], which is a powerful tool for investigating the turning point and stability. Unlike this method, our approach is based on the extended phase space thermodynamics and on the slope of $T$ versus $S$ as a primitive equation for obtaining the dynamical pressure, where its maximum is related to a critical point and possible phase transition.

The layout of the paper is as follows: In Sec. [II we give a brief review of the canonical ensemble approach and the van der Waals approach to black hole phase transitions. In Sec. III we introduce an alternative scheme for calculating the phase transition parameters, and we employ this method to the usual van der Waals system. In Sec. IV] we perform a case study of the Reissner-Nordström AdS black hole to elaborate the method. Finally, we make several remarks in the conclusion.

\section{BRIEF REVIEW OF VARIOUS METHODS}

\section{A. The usual method: heat capacity and extended phase space}

In the canonical ensemble, discontinuities of the heat capacity are indicated as the phase transition points. The heat capacity in the context of canonical ensemble is given by

$$
C_{Q}=\frac{T}{\left(\frac{\partial^{2} M}{\partial S^{2}}\right)_{Q}}=T\left(\frac{\partial S}{\partial T}\right)_{Q}
$$

The main application of heat capacity is for studying thermal stability. Positivity of $C_{Q}$ can guarantee thermal stability of a system, while its negativity is regarded as an instability.

On the other hand, in order to study the critical behavior of a thermodynamical system, one is required to obtain an equation of state, $P=P(T, V)$. In the context of black hole thermodynamics, one may find the temperature of a typical black hole in the presence of the cosmological constant $T=T\left(m, r_{+}, \Lambda, Q\right.$, other hairs $)$. We can consider the cosmological constant as a dynamical pressure and take into account the relation between the event horizon radius $\left(r_{+}\right)$and the volume to find an equation of state, $P=P(T, V)$. Applying the properties of a critical point in an isothermal $P-V$ diagram (inflection point), one finds $\left(\frac{\partial P}{\partial V}\right)_{T}=\left(\frac{\partial^{2} P}{\partial V^{2}}\right)_{T}=0$. This relation helps us to find the critical points, and the possible phase transitions. This method very much depends on the value of temperature. It is worth mentioning that this method is not practical for black holes with nonspherical horizon in most gravitational theories. In order to avoid such a restriction, we should use an alternative method for obtaining the critical values in the extended phase space.

\section{B. van der Waals liquid-gas system (a brief review)}

The van der Waals system is one of the important models for describing a real liquid-gas system and its critical behavior. The equation of state of this model is a modification of the ideal gas equation, and considers the non-zero size of the molecules and the attraction between them. The van der Waals equation of state is given by [18]

$$
\left(P+\frac{a}{v^{2}}\right)(v-b)=k T(P, v),
$$

where $P$ and $T$ are the pressure and temperature, respectively. Also, $v$ is the specific volume $v=\frac{V}{N}, b$ is a free parameter related to the non-zero size of the molecules of a fluid, and $a$ represents the strength of attraction between the molecules. Here, $k$ is a constant that can be set to one without loss of generality. Note that setting $a=b=0$ yields the familiar ideal gas law. Due to the van der Waals-black hole correspondence, one can use the analogy between the temperature (and hence equation of state) of the fluid and the temperature of the black hole. The existence of critical behavior can be determined by examining the properties of the inflection point, which satisfy

$$
\left(\frac{\partial P}{\partial v}\right)_{T}=\left(\frac{\partial^{2} P}{\partial v^{2}}\right)_{T}=0
$$

Using the inflection point of the equation of state of the van der Waals system Eq. (2), it is straightforward to find the following critical values

$$
v_{c}=3 b, \quad \& \quad P_{c}=\frac{a}{27 b^{2}}, \quad \& \quad T_{c}=\frac{8 a}{27 b k} .
$$

Inserting $T=T_{c}$ in the equation of state, we find two inseparable phases of liquid-gas with a possible phase transition between them. For the case $T<T_{c}$, we have a phase transition between two phases of liquid and gas. However, there exists a region of specific volume in which no physical system exists and the phase transition takes place over it. In other words, for this case, two specific volumes with the same pressure exist, and the phase transition takes place between them. In order to obtain all of these critical behaviors and their specific critical values, one has to take into account all temperatures smaller than $T_{c}$, which is practically impossible. Our method, introduced in this paper, provides the possibility of obtaining all of these critical points analytically. We will demonstrate this possibility in what follows.

The Gibbs free energy of this system can be expressed in the following form

$$
G=-k T\left(1+\ln \left[\frac{v-b}{\Phi} T^{\frac{3}{2}}\right]\right)-\frac{a}{v}+P v
$$

where $\Phi$ is a constant characterizing the gas. The entropy of the system can be obtained from the differential 
equation $d G=-S d T+v d P$, which leads to

$$
S=k\left(\frac{5}{2}+\ln \left[\frac{v-b}{\Phi} T^{\frac{3}{2}}\right]\right) .
$$

Using the equation of state and inserting the temperature in Eq. (6), one can find the following $S(v, P)$

$$
S(v, P)=k\left(\frac{5}{2}+\ln \left[\frac{(v-b)^{\frac{5}{2}}\left(P+\frac{a}{v^{2}}\right)^{\frac{3}{2}}}{k^{\frac{3}{2}} \Phi}\right]\right) .
$$

In order to use the method introduced in this paper, one needs the enthalpy of the system. This quantity can be calculated using different methods, and the following relation is one of them

$$
H=G+T S=\frac{3}{2} k T-\frac{a}{v}+P v,
$$

which is known as the enthalpy of the van der Waals system. Using the equation of state, it is easy to find the following relation for $H(v, P)$

$$
H(v, P)=\left(\frac{5 v-3 b}{2}\right) P+\frac{a(v-3 b)}{2 v^{2}},
$$

where, in principle, one may remove $v$ from Eqs. (7) and (9) to obtain $H=H(S, P)$.

\section{ALTERNATIVE APPROACH TO PHASE TRANSITIONS}

Taking into account the postulates of the usual thermodynamics, it appears that all complete differentiations can be written as functions of three thermodynamic variables. It is known that these three variables are not independent, for instance, an equation of state can reduce the number of degrees of freedom to two. On the other hand, the equations for the thermodynamical properties of the system are combinations of different variables. For example, in most cases, pressure and temperature, Gibbs free energy and internal energy, etc., of a thermodynamical system are not independent of each other. Pressure and temperature are related by the equation of state, and the Gibbs free energy may be derived by the Legendre transform of the internal energy. Hence, internal and Gibbs free energies, as pressure and temperature, are dependent on each other.

In practice, obtaining all possible critical points of a system, as well as the ranges in which phase transitions take place, is not possible with the usual methods. The main reason is that one has to take the value of a particular thermodynamical quantity smaller than its critical value to obtain the critical points and their corresponding range of phase transitions. Mathematically, it is not possible to solve such a problem analytically using the usual methods. We would like to introduce here an alternative method that provides such a possibility, and which results in new relations between thermodynamical quantities. These relations provide information about the phase transitions of a system and their corresponding ranges.

Since divergence points of the heat capacity $\left(\left(\frac{\partial S}{\partial T}\right)_{P}=\right.$ $\left.\frac{1}{\left(\frac{\partial T}{\partial S}\right)_{P}} \rightarrow \infty\right)$ hint us for the possible phase transition (see Eq. (1)), the equation of vanishing slope of $T$ versus $S$ helps us to obtain a new relation for pressure (and look for phase transition by maximizing the new relation for pressure). We should note that in order to find the critical behavior of a system, the vanishing slope of $T$ versus $S$ is necessary but not sufficient in our approach. The method is as follows: instead of considering the usual equation of state, we use the equation of vanishing slope of $T$ versus $S,\left(\frac{\partial T}{\partial S}\right)_{P}=0$. This equation is solved with respect to pressure in the extended phase space. This leads to a new relation for the pressure which is only volume dependent. This relation differs from the other relations which are obtained using the usual equation of state. The existence of a maximum of pressure from this relation must be examined. The maximum(maxima) of this relation is(are) the critical point(s) where the phase transition takes place. In other words, the maximum of this relation is where the system undergoes a phase transition.

It is evident that by finding a maximum, one is able to extract the critical pressure and the horizon radius (volume) at the same time. It is worth mentioning that to obtain the relation for pressure one can use $\left(\frac{\partial^{2} H}{\partial S^{2}}\right)_{P}$ instead of $\left(\frac{\partial T}{\partial S}\right)_{P}$, where $H$ is the enthalpy of the system. The maximum is where the system acquires a phase transition (the maximum indicates the critical values for a system). On the other hand, thermodynamical concepts indicate that for pressures smaller than the critical one, two critical volumes exist between which a phase transition takes place. For pressures larger than the critical pressure, no phase transition exists. We see that due to the existence of critical pressure at the maximum of this relation, such a property is preserved in our method, and all possible critical points and their corresponding ranges are obtained. Furthermore, using the new relation for pressure, and replacing it in the usual equation of state, one can obtain a new relation for temperature, which is free of pressure. The same can be done for enthalpy, internal energy or Gibbs free energy, which leads to new relations which are only volume dependent. In order to elaborate the efficiency of the presented method, we give in what follows two typical but general examples in the context of the usual thermodynamics and black hole thermodynamics.

\section{A. The case of van der Waals liquid/gas system}

We are now in a position to use our method for the case of van der Waals system. Since both the entropy $S(v)$ and enthalpy $H(v)$ are volume dependent at constant 
pressure (see Eqs. (7) and (9)), we can use the following relation

$$
\left(\frac{\partial^{2} H}{\partial S^{2}}\right)_{P}=\left(\frac{\partial S}{\partial v}\right)_{P}^{-1} \frac{\partial}{\partial v}\left[\left(\frac{\partial H}{\partial v}\right)_{P}\left(\frac{\partial S}{\partial v}\right)_{P}^{-1}\right]_{P},
$$

which leads to the following relation for a van der Waals liquid/gas system

$$
\left(\frac{\partial^{2} H}{\partial S^{2}}\right)_{P}=\frac{2(v-b)\left(P+\frac{a}{v^{2}}\right)\left(P v^{3}-a v+2 a b\right)}{k^{2}\left(5 P v^{3}-a v+6 a b\right)} .
$$

From the above relation, one finds that enthalpy changes during a phase transition. In other words, the increasing/decreasing behavior of enthalpy is different before and after a phase transition, and also before and after a critical point. By solving $\left(\frac{\partial^{2} H}{\partial S^{2}}\right)_{P}=0$ with respect to $P$, one can get the following new relation for pressure, which differs from the usual equation of state

$P_{\text {new }}=\left\{\begin{array}{ll}\frac{v-2 b}{v^{3}} a, & \text { allowed } \\ -\frac{a}{v^{2}}, & \text { unallowed, due to the equation of state }\end{array}\right.$.

Using the concept of extremum of this (allowed) relation, that is the critical point, one finds following critical volume and pressure

$$
v_{c}=3 b, \quad \& \quad P_{c}=\frac{a}{27 b^{2}},
$$

which are identical to those obtained previously in Eq. (44). By replacing the new pressure in the equation of state, Gibbs free energy and enthalpy, one can get new relations for these thermodynamical quantities (as well as for other), which are pressure independent

$$
\begin{aligned}
T_{\text {new }} & =\frac{2 a(b-v)^{2}}{k v^{3}}, \\
G_{\text {new }} & =-k T\left(1+2 \ln \left[\frac{v-b}{\Phi} T^{\frac{3}{2}}\right]\right)-\frac{2 a b}{v}, \\
H_{\text {new }} & =\frac{3}{2} k T-\frac{2 a b}{v^{2}} .
\end{aligned}
$$

These new relations enable us to extract all possible critical temperatures, Gibbs free energy and enthalpy that a system can acquire. In order to highlight this aspect of our method, we use the plot in Fig. 1.

It is evident that the maxima of the new relations for the temperature $T_{n e w}$ and pressure $P_{n e w}$ are where the system undergoes a phase transition. Note that in the $P-v$ picture, the temperature is kept constant, while in the $T-v$ picture, the pressure is kept constant. In addition, for pressures (temperatures) smaller than the critical pressure (temperature), the new relation gives a single pressure (temperature) with two related volumes.
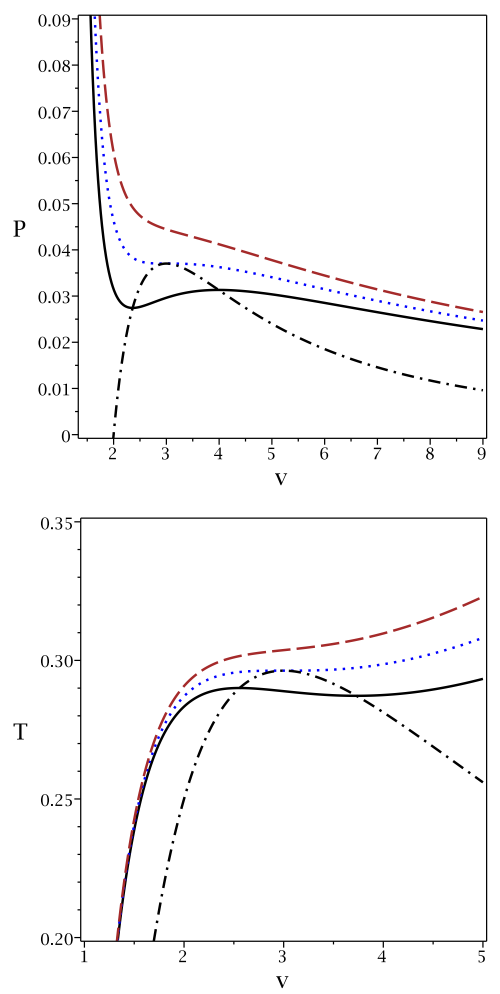

FIG. 1: (color online) Application of the method to the case of the van der Waals system. Top panel: $P_{\text {new }}$ (dash-dotted line) and $P$ versus $v$ for $T=0.9 T_{c}$ (continuous line), $T=T_{c}$ (dotted line) and $T=1.1 T_{c}$ (dashed line).

Bottom panel: $T_{\text {new }}$ (dash-dotted line) and $T$ versus $v$ for $P=0.9 P_{c}$ (continuous line), $P=P_{c}$ (dotted line) and $P=$ $1.1 P_{c}$ (dashed line). In both diagrams, we chose $b=1, a=4$ and $k=1$.

The phase transition takes place between these two volumes at a specific pressure. As one can see, all possible critical points and the corresponding ranges of phase transitions are included in this method. This is one of the important features of our method which was not possible with previous methods. It is interesting to note that the minima of $T(P)$ coincide with the maxima of the $T_{\text {new }}\left(P_{\text {new }}\right)$. We continue with another example in the context of black holes.

\section{PHASE TRANSITION IN HIGHER DIMENSIONAL REISSNER-NORDSTRÖM ADS BLACK HOLE}

The main motivation to study asymptotically AdS black holes stems from the hypothesis of AdS/CFT correspondence. Using the thermal field theoretic approaches, it has been deduced that AdS black holes undergo certain phase transitions. The first sign of such phase transitions was observed by Page and Hawking for the Schwarzschild AdS black hole 19]. With the addition of parameters such as electric charge and spin, the phase transition 
process is more elaborate and enhanced. It is quite interesting to note that the pressure-volume picture of the ideal gas for constant temperature is also mimicked by the AdS black holes (see Fig.1 top panel). This analogy between a gravitational system (an AdS black hole) and a non-gravitational thermal system (such as an ideal gas or a van der Waals fluid) is established by identifying a correspondence between their parameters i.e. mass with enthalpy, temperature with surface gravity, entropy with area, and cosmological constant with pressure. Thus, in the first law of thermodynamics, the cosmological constant appears as pressure, which is conjugate to the volume of a black hole [20]. Besides, using the reverse isoperimetric inequality, it has been deduced that entropy inside the horizon of a given volume is maximized for the Schwarzschild AdS black hole [21].

In the black hole systems, it was shown that one can take the negative cosmological constant as thermodynamical pressure [22] with the following relation

$$
P=-\frac{\Lambda}{8 \pi}
$$

On a complementary note, we should mention that for specific black holes in modified general relativity, such as dilatonic gravity and gravity's rainbow, one has to use a modified proportionality relation instead of Eq. (14) [23, 24]. Although in this paper we consider a well-known Reissner-Nordström AdS black hole, our technique is consistent with the other black holes in modified theories of gravity. Replacing the cosmological constant with thermodynamical pressure (working in the extended phase space thermodynamics) leads to the following important results:

I) The resulting temperature for the black hole is the equation of state.

II) The total mass of the black hole is no longer the internal energy. In fact, it is replaced by the enthalpy in such considerations. This results in the following relation for the Gibbs free energy

$$
G=M-T S
$$

We would like to give now an example of the validity of our approach and its consistency with previous methods in the context of black hole systems. For this purpose, we study the critical behavior of the $d$-dimensional Reissner-Nordström AdS black hole. Previously, the results for this specific black hole were derived using the usual method in Ref. [18]. The metric of this black hole in spherically symmetric spacetime is given as

$$
d s^{2}=-\psi(r) d t^{2}+\frac{d r^{2}}{\psi(r)}+r^{2} d \Omega_{d_{2}}^{2}
$$

where we use the notation $d_{i}=d-i$. In Eq. (16),$d \Omega_{d_{2}}^{2}$ denotes the metric of $\left(d_{2}\right)$-dimensional unit sphere, and

$$
\psi(r)=1-\frac{m}{r^{d_{3}}}-\frac{2 \Lambda}{d_{1} d_{2}} r^{2}+\frac{2 d_{3} q^{2}}{d_{2} r^{2 d_{3}}} .
$$

The temperature, entropy and total finite mass of this black hole are calculated using the surface gravity, area law and ADM approach, respectively, which lead to

$$
\begin{aligned}
T & =\frac{d_{3}}{4 \pi r_{+}}-\frac{\Lambda r_{+}}{2 \pi d_{2}}-\frac{d_{3}^{2} q^{2}}{2 \pi d_{2} r_{+}^{2 d_{5 / 2}}}, \\
S & =\frac{r_{+}^{d_{2}}}{4} \\
M & =\frac{d_{2}}{16 \pi} m
\end{aligned}
$$

where $r_{+}$is the outer horizon of the black hole. By evaluating the metric function on the outer horizon $\left(\psi\left(r=r_{+}\right)=0\right)$, we obtain

$$
M=\frac{d_{2}}{16 \pi}\left(r_{+}^{d_{3}}-\frac{2 r_{+}^{d_{1}}}{d_{1} d_{2}} \Lambda+\frac{2 d_{3} q^{2}}{d_{2} r_{+}^{d_{3}}}\right)
$$

with the following relation for the Gibbs free energy

$$
G=\frac{r_{+}^{d_{3}}}{16 \pi}+\frac{d_{3}(2 d-5) q^{2}}{8 \pi d_{2} r_{+}^{d_{3}}}-\frac{r_{+}^{d_{1}}}{d_{1} d_{2}} P
$$

Another interesting method for calculating the thermodynamic potentials (such as the Gibbs free energy) in a gravitational system is based on the Euclidean onshell action. Since bulk action of the theory diverges, we use the counter-term action (as a boundary action) to remove the divergency. In addition, we should add the Gibbons-Hawking and electromagnetic boundary terms to the bulk action to get a well-defined action. The wellbehaved finite action can be written as (see [26])

$I=I_{b}+I_{c t}-\frac{1}{8 \pi} \int_{\partial M} d^{n} x \sqrt{\gamma} K-\frac{1}{4 \pi} \int_{\partial M} d^{n} x \sqrt{\gamma} n_{\mu} F^{\mu \nu} A_{\nu}$

where $I_{b}$ and $I_{c t}$ are, respectively, the bulk and counterterm actions of the Einstein-Maxwell gravity. Also, $\gamma_{i j}$ and $K$ are, respectively, the induced metric and the extrinsic curvature of the boundary. Using Eq. (23), it is straightforward to calculate the total on-shell action with respect to the volume of the unit $d_{2}-$ sphere

$$
I=\frac{\beta}{16 \pi}\left(r_{+}^{d_{3}}+\frac{2 \Lambda r_{+}^{d_{1}}}{d_{1} d_{2}}+\frac{2 d_{3}(2 d-5) q^{2}}{d_{2} r_{+}^{d_{3}}}\right)
$$

where $\beta$ is the inverse of the Hawking temperature. Using the fact that $G=I / \beta$, with Eq. (14) we find

$$
G=\frac{1}{16 \pi}\left(r_{+}^{d_{3}}-\frac{16 \pi P r_{+}^{d_{1}}}{d_{1} d_{2}}+\frac{2 d_{3}(2 d-5) q^{2}}{d_{2} r_{+}^{d_{3}}}\right)
$$

which is the same as Eq. (22), as expected.

We are now in a position to calculate the critical values with the usual method. First, we calculate the volume conjugate to the pressure as

$$
V=\left(\frac{\partial H}{\partial P}\right)_{S, Q}=\left(\frac{\partial M}{\partial P}\right)_{S, Q}=\frac{r_{+}^{d_{1}}}{d_{1}} .
$$


Since the volume depends on the horizon radius, one can use the horizon radius for investigating the thermodynamic behavior of the black hole, proportional (linearly) to its specific volume [18]. Using Eqs. (14) and (18), one obtains the equation of state as

$$
P=\frac{d_{2}\left(4 \pi T r_{+}^{3}-d_{3} r_{+}^{2}\right)}{16 \pi r_{+}^{4}}+\frac{2 d_{3}^{2} q^{2} r_{+}^{-2 d_{4}}}{16 \pi r_{+}^{4}} .
$$

We now employ the proprieties of the inflection point, $\left(\frac{\partial P}{\partial r_{+}}\right)_{T}=\left(\frac{\partial^{2} P}{\partial r_{+}^{2}}\right)_{T}=0$, to obtain the critical horizon radius (volume) $r_{c}$, which leads to

$$
r_{c}^{2}-4 d_{3} d_{5 / 2} q^{2} r_{c}^{-2 d_{4}}=0
$$

with the following solution [18]

$$
r_{c}=\left(4 q^{2} d_{3} d_{5 / 2}\right)^{\frac{1}{2 d_{3}}} .
$$

It is a matter of calculation to obtain the critical temperature and pressure as

$$
\begin{aligned}
& T_{c}=\frac{d_{3}}{2 \pi\left(4 q^{2} d_{3} d_{5 / 2}\right)^{\frac{1}{2 d_{3}}}}-\frac{\left(4 q^{2} d_{3} d_{5 / 2}\right)^{\frac{d_{5 / 2}}{d_{3}}} d_{3}^{2} q^{2}}{\pi} \\
& P_{c}=\frac{d_{2} d_{3}}{16 \pi\left(4 q^{2} d_{3} d_{5 / 2}\right)^{\frac{1}{d_{3}}}}-\frac{d_{5 / 2} d_{3}^{2} q^{2}}{4 \pi\left(4 q^{2} d_{3} d_{5 / 2}\right)^{\frac{d_{2}}{d_{3}}}} .
\end{aligned}
$$

Let us now get the critical values using our approach. Using Eqs. (19) and (21), and replacing the cosmological constant with the pressure in Eq. (14), one can obtain (since at constant pressure both $S=S\left(r_{+}\right)$and $M=M\left(r_{+}\right)$are independent of the temperature, it is not needed to use the equation of state for removing $T$, which was used before in the van der Waals liquid/gas system)

$$
\left(\frac{\partial^{2} M}{\partial S^{2}}\right)_{q, P}=\frac{16 P}{d_{2}^{2} r_{+}^{d_{3}}}-\frac{d_{3}}{\pi d_{2} r_{+}^{d_{1}}}+\frac{4 d_{5 / 2} d_{3}^{2} q^{2}}{\pi d_{2}^{2} r_{+}^{3 d_{7 / 3}}} .
$$

Solving $\left(\frac{\partial^{2} M}{\partial S^{2}}\right)_{q, P}=0$ with respect to $P$, we obtain a new relation for the pressure

$$
P_{\text {new }}=\frac{d_{2} d_{3}}{16 \pi r_{+}^{2}}-\frac{d_{5 / 2} d_{3}^{2} q^{2}}{4 \pi r_{+}^{2 d_{2}}} .
$$

Replacing the pressure in the relations for temperature Eq. (18), mass Eq. (21) and Gibbs free energy Eq. (22) with the new pressure relation (33), one can obtain new relations for these thermodynamical quantities in following form

$$
\begin{aligned}
T_{\text {new }} & =\frac{d_{3}}{2 \pi r_{+}}-\frac{d_{3}^{2} r_{+}^{5-2 d} q^{2}}{\pi}, \\
M_{\text {new }} & =\frac{d_{2}^{2} r_{+}^{d_{3}}}{8 \pi d_{1}}-\frac{d_{2} d_{3} d_{4} q^{2}}{4 \pi d_{1} r_{+}^{d_{3}}} \\
G_{\text {new }} & =\frac{r_{+}^{d_{3}}}{8 \pi d_{1}}+\frac{d_{5 / 2} d_{3} q^{2}}{2 \pi d_{1} r_{+}^{d_{3}}} .
\end{aligned}
$$
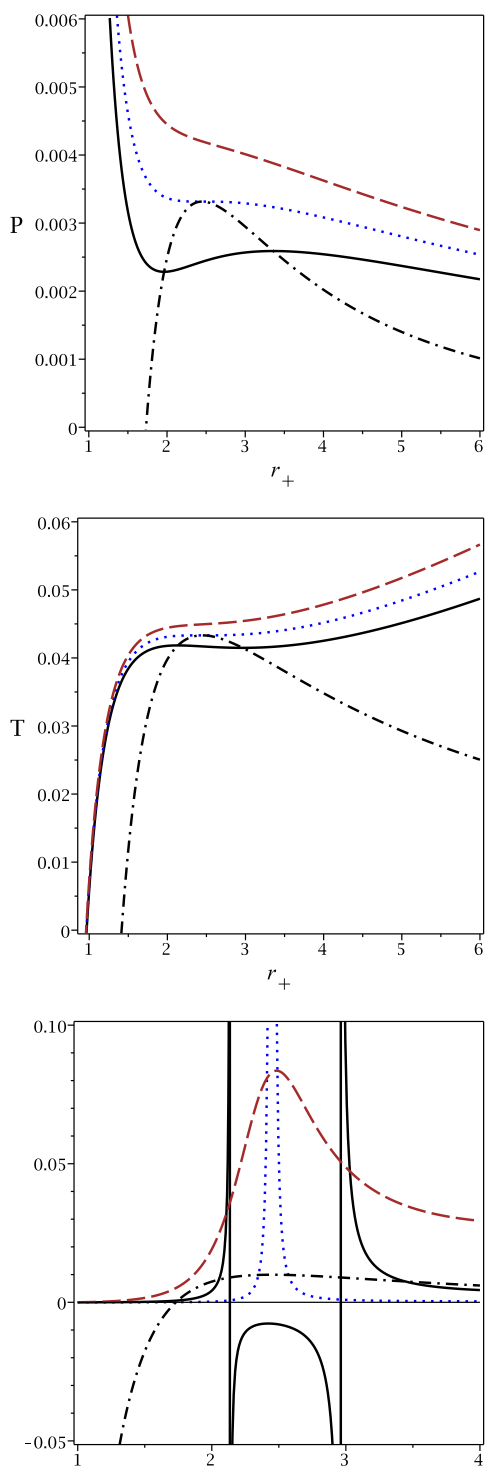

FIG. 2: (color online) Top panel: $P_{\text {new }}$ (dash-dotted line) and $P$ versus $r_{+}$, for sub-critical case $T=0.9 T_{c}$ (continuous line), critical case $T=T_{c}$ (dotted line) and super-critical case $T=1.1 T_{c}$ (dashed line).

Middle panel: $T_{\text {new }}$ (dash-dotted line) and $T$ versus $r_{+}$, for sub-critical $P=0.9 P_{c}$ (continuous line), critical $P=P_{c}$ (dotted line) and super-critical $P=1.1 P_{c}$ (dashed line).

Bottom panel: $P_{\text {new }}$ (dash-dotted line) and $C_{Q}$ versus $r_{+}$, for sub-critical $P=0.9 P_{c}$ (continuous line), critical $P=P_{c}$ (dotted line) and super-critical $P=1.1 P_{c}$ (dashed line). In all three panels, $q=1$ and $d=4$.

It is evident that the relation for pressure Eq. (33) is different from the usual equation of state Eq. (27). In order to obtain the maximum of this relation, we use the 
mathematical nature of the extremum

$$
\left.\left(\frac{d P_{\text {new }}}{d r_{+}}\right)\right|_{r_{+}=r_{N C}}=\frac{d_{2} d_{3}^{2} d_{5 / 2} q^{2}}{2 \pi r_{N C}^{2 d_{3 / 2}}}-\frac{d_{2} d_{3}}{8 \pi r_{N C}^{3}}=0
$$

with the following solution for the new critical (NC) horizon radius, $r_{N C}$

$$
r_{N C}=\left(4 d_{3} d_{5 / 2} q^{2}\right)^{\frac{1}{2 d_{3}}},
$$

which is exactly the same as that obtained in Eq. (29). Replacing this horizon radius in Eqs. (34) and (33), it is a matter of calculation to find

$$
\begin{aligned}
& T_{N C}=\frac{d_{3}}{2 \pi\left(4 q^{2} d_{3} d_{5 / 2}\right)^{\frac{1}{2 d_{3}}}}-\frac{\left(4 q^{2} d_{3} d_{5 / 2}\right)^{\frac{d_{5 / 2}}{d_{3}}} d_{3}^{2} q^{2}}{\pi}(39) \\
& P_{N C}=\frac{d_{2} d_{3}}{16 \pi\left(4 q^{2} d_{3} d_{5 / 2}\right)^{\frac{1}{d_{3}}}}-\frac{d_{5 / 2} d_{3}^{2} q^{2}}{4 \pi\left(4 q^{2} d_{3} d_{5 / 2}\right)^{\frac{d_{2}}{d_{3}}}}, \quad(40)
\end{aligned}
$$

which are exactly the same as the previously calculated critical temperature Eq. (30) and pressure Eq. (31). These results show that the critical values calculated in our approach are consistent with those calculated with the usual method in extended phase space.

Using the critical radius Eq. (38) with the new relations for the mass Eq. (35) and Gibbs free energy Eq. (36), we obtain the critical mass (enthalpy) and critical Gibbs free energy as well

$$
\begin{aligned}
M_{N C} & =\frac{d_{2} d_{3}\left(d^{2}-5 d+7\right) q}{4 \pi d_{1} \sqrt{d_{3} d_{5 / 2}}}, \\
G_{N C} & =\frac{\sqrt{d_{3} d_{5 / 2}} q}{2 \pi d_{1}} .
\end{aligned}
$$

In order to elaborate the results of our approach in more detail, we plot in Fig. 2 the obtained relations for temperature and pressure. It is clear that for pressures larger than the critical one (the temperature is larger than its critical value in the van der Waals-like diagram), no phase transition is observed for the van der Waals-like diagram (dashed line of Fig. 2, top and middle panels). If no phase transition occurs in a black hole, this implies that the black hole remains physically intact, i.e. its mass and the other physical parameters remain the same. The black hole remains stable and does not radiate thermally. This may correspond to a state of thermal equilibrium. If the equilibrium gets unstable, the heat capacity of the black hole become negative, causing the black hole to radiate. In this case, a phase transition does take place. Similarly for same conditions, no critical pressure is observed in our approach. On the contrary, for pressures smaller than the critical value (the temperature is smaller than the critical temperature), two critical horizons are observed for any pressure (temperature) which is in agreement with the results of our approach (continuous line in Fig. 2, top and middle panels). Finally, we observe that the critical pressure (critical temperature) and the critical horizon radius calculated by the usual method, coincide with the maximum of the new relation for pressure and its related horizon radius. This also indicates that the results of our method, are completely in agreement with the previous method.

Finally, we plot the heat capacity (bottom panel in Fig. (2) to show the consistency of the new pressure. It is evident that for $P<P_{c}$, two points of discontinuity exist for the heat capacity, which are coincident with the phase transition points that are observed in the other methods. If the pressure is equal to the critical pressure, only one discontinuity is observed in the heat capacity, as in the other methods. For $P>P_{c}$, no discontinuity is observed for the heat capacity. This behavior indicates that all methods give consistent results.

In the top panel of Fig. 2, one can see the so-called saturation curve, dash-dotted line. Taking into account the $P-V$ isothermal diagram with $T<T_{c}$ (continuous line), we can decrease the horizon radius to find two points of intersection with the saturation curve $\left(r_{+1}\right.$ and $r_{+2}$ with $\left.r_{+1}<r_{+2}\right)$. The black hole system is unstable for $r_{+1}<r_{+}<r_{+2}$. In other words, there is a phase transition between a small and a large black hole (between $r_{+1}$ and $r_{+2}$ ). This phase transition may occur with a sudden burst of thermal Hawking radiations, i.e. the size of the black hole suddenly shrinks from $r_{+2}$ to $r_{+1}$ without changing the black hole temperature. Black hole solutions are not physical between these two points. This can be explained by the fact that the heat capacity is negative (see bottom panel of Fig. 2 for more detail), and also by the fact that the speed of sound is larger than the speed of light [25]. Note that similar discontinuities in specific heat capacity also occur in the Born-Infeld black holes 11]. It is worth noting that for $T=T_{c}$, the intersection points meet and are equal to the critical horizon radius $r_{+1}=r_{+2}=r_{c}$. The same statement could be made for the temperature in the middle panel of Fig. 2 ,

Before finishing the paper, it is worth pointing out the significance of our approach.

First of all, our method provides the possibility of obtaining different thermodynamical quantities independent of each other. In other words, as one can see from Eqs. (34)-(36), they only depend on such properties as dimension, electric charge and horizon radius. If we generalize the action to other gravitational theories or include other matter fields, the resultant new temperature, pressure, mass and Gibbs free energy obtained using our method, have the same properties (they are only a function of black hole properties).

Second, the new relations include only critical points that a black hole could acquire in different conditions. In the usual methods, in order to get all points between which phase transitions take place, one has to consider pressures which are equal or smaller than the critical pressure. Technically, such a task is impossible if it is to be done for all pressures. Using our method, one can find all possible phase transitions, horizon radii and cor- 
responding pressures that a system could acquire. The same could be said for the new relations for temperature, mass and Gibbs free energy. In other words, by using our approach, one can obtain all phase transition points and corresponding critical temperature, pressure, mass and Gibbs free energy that system can acquire in analytical form.

Third, using our method, one can get the range of horizon radii that depends on the critical values, in which the black hole solutions do not exist. For clarification, one should take a look at the diagram for the new pressure in the top panel of Fig. 2 (dashed-dotted line). Clearly, the phase transition takes place between two points with the same pressure. The prohibited range of horizon radii for the black hole is between these points. Taking a closer look, one can see that by using our approach, one can obtain the maximum range of the horizon radii in which the black hole solutions do not exist. Such a maximum could not be obtained easily with the usual methods. In addition, by using our approach, one can get the rate of increase of the prohibited range of horizon radii by studying the behavior of its diagram. Such a procedure may encounter significant problems for the usual method. Finally, we should point out that these three features are also valid for the usual thermodynamical systems.

\section{CLOSING REMARKS}

Motivated by the interest in van der Waals-like behavior and recent progress in the thermodynamic phase transitions of black holes, we introduced an alternative approach for studying the phase transition points in both the usual thermodynamical systems and black holes.

Although the usual method of studying a phase transition originates in the temperature as the equation of state, our approach is based on the slope of temperature versus entropy, and is a powerful method to address the critical behavior of a thermodynamical system.

The results of our approach are in agreement with the other methods. However, it also provides further infor- mation regarding the critical behavior of thermodynamical systems, which could not be derived with the other methods. As the highlights of our method, one can state: I) Obtaining new relations for different thermodynamical quantities which are independent of each other. II) Mapping all possible critical points and regions in which phase transitions take place.

Since it is known that the four-dimensional ReissnerNordström black hole has the reentrant phase transition, it would be interesting to examine if our method confirms (or not) the existence of the reentrant phase transition. The method introduced here is applicable to both the usual thermodynamical systems and black holes. This shows that one can also employ the general structure of this method in the context of other physical systems such superconductors, condense matter systems, gauge/gravity duality, or even in the context of quantum systems. It is also interesting to build a geometrical theory based on the Legendre invariance, like the known theory of geometrothermodynamics, or on other types of symmetries. As a future task, it would be interesting to extend the present study of phase transitions and critical phenomenon via AdS/CFT [27]. Furthermore, how do phase transitions alter the geometry and topology of black holes, is a question for a separate investigation. We also plan to investigate the relationship between the cosmic censorship hypothesis and the various phase transition of black holes, and to work on phase transitions and critical behavior of black rings, black saturns and black membranes.

\section{Acknowledgements}

We thank an anonymous referee for useful comments. We also thank the editor of Chinese Physics C for improving the text and presentation. We wish to thank the Shiraz University Research Council. This work has been supported financially by Research Institute for Astronomy and Astrophysics of Maragha (RIAAM), Iran.
[1] D. Y. Lando and V. B. Teif, J. Biomol. Struct. Dynam. 17, 903 (2000).

[2] G. Longo, and M. Montevil, Progress Biophys. Molec. Biology, 106, 340 (2011).

[3] H. Kleinert, Phys. Rev. D 60, 085001 (1999).

[4] A. L. Greer, Science, 267, 1947 (1995).

[5] K. Kumar, A. K. Pramanik, A. Banerjee, P. Chaddah, S. B. Roy, S. Park, C. L. Zhang and S. W. Cheong, Phys. Rev. B 73, 184435 (2006).

[6] G. Tarjus, Nature 448, 758 (2007).

[7] H. B. Callen, "Thermodynamics and an Introduction to Thermostatistics" (John Wiley \& Sons, Inc., New York, 1985).

[8] D. Layzer, "Cosmogenesis, The Development of Order in the Universe", (Oxford Univ. Press, 1991).
[9] D. C. Zou, Y. Liu and R. Yue, Eur. Phys. J. C 77, 365 (2017).

[10] G. J. Stephens and B. L. Hu, Int. J. Theor. Phys. 40, 2183 (2001).

[11] D. Roychowdhury, arXiv:1403.4356.

[12] V. Niarchos, Mod. Phys. Lett. A 23, 2625 (2008).

[13] T. Harmark, N. A. Obers, [arXiv: hep-th/0503020].

[14] H. Poincare, Acta. Math. 7, 259 (1885).

[15] R. D. Sorkin, Astrophys. J. 257, 847 (1982).

[16] O. Kaburaki, I. Okamoto and J. Katz, Phys. Rev. D 47, 2234 (1993).

[17] M. Azreg-Aïnou and M. E. Rodrigues, JHEP 09, 146 (2013).

[18] D. Kubiznak and R. B. Mann, JHEP 07, 033 (2012).

[19] S. W. Hawking and D. N. Page, Commun. Math. Phys. 
87, 577 (1983).

[20] A. Rajagopal, D. Kubiznak and R. B. Mann, Phys. Lett. B 737, 277 (2014).

[21] M. Cvetic, G. W. Gibbons, D. Kubiznak and C. N. Pope, Phys. Rev. D 84, 024037 (2011).

[22] B. P. Dolan, Class. Quantum Gravit. 28, 125020 (2011).

[23] S. H. Hendi, M. Faizal, B. Eslam Panah and S. Panahiyan, Eur. Phys. J. C 76, 296 (2016).

[24] S. H. Hendi, S. Panahiyan, B. Eslam Panah, M. Faizal and M. Momennia, Phys. Rev. D 94, 024028 (2016).

[25] S. H. Hendi, N. Riazi and S. Panahiyan, Ann. Phys. (Berlin) 530, 1700211 (2018).

[26] M. H. Dehghani, Ch. Shakuri and M. H. Vahidinia, Phys. Rev. D 87, 084013 (2013).

[27] M. Natsuume, Prog. Theor. Phys. Suppl. 186, 491 (2010). 